\title{
8. Innovation of Chinese listed enterprises: Evaluation and policies ${ }^{1}$
}

\section{Zhongyu Ma, Huiqing Gao, Weihua Yin and Zhichao Wen}

At the end of 2012, the report of the eighteenth National Congress of the Communist Party of China proposed that, by 2020, China would become one of the most innovative countries in the world. This was the first time such a goal had been set since China's period of reform and opening-up began; it was also the first time in the thousands of years of China's history.

Over the years, the Chinese Government has carried out reforms designed to delegate power to enterprises, streamline administration and optimise government services, to promote large-scale entrepreneurship and encourage cultural and educational innovation. As a result, an atmosphere of innovation has taken hold in Chinese society; the innovation system has been continuously improved, levels of innovation have been growing and new modes of business have emerged. According to the 2018 Global Innovation Index, published by the World Intellectual Property Organization (WIPO), Cornell University and other institutions, mainland China has moved up five positions to seventeenth place since 2017. This is the first time mainland China has featured in the top 20-signalling that it is becoming one of the world's most innovative economies.

Behind the rapid improvement of China's overall innovative competency is the improvement of the innovative competency of Chinese companies, with listed companies the main force of company innovation and development. With the help of 'big data' technology, China has built a system to evaluate technological and model innovation competency, which is the first integrated system anywhere that integrates technological and model innovation competency in one index. The results of the evaluation show that, although China's national innovation competency is ranked among the top 20 in the world, Chinese listed companies are generally in the preliminary stages of innovation and development, and the potential for future progress is great.

It can be foreseen that, with the continuous implementation of innovation-driven strategies, Chinese companies will gradually become the main body driving innovation, and the national science and technological innovation system will be greatly improved. In addition, the goal of China becoming one of the most innovative countries in the world by 2030 , as well as establishing a world-class innovation centre by 2050 , can be achieved.

1 Based on the data of listed enterprises in 2017. 


\section{Theories and methods}

Drawing on previous theoretical findings and considering the characteristics of innovation, we propose a method to establish the first index evaluation system for listed companies' technological and model innovation competency.

\section{Relevant theories of evaluation of listed companies' innovation}

\section{Two basic theories}

\section{Theory of innovation ecology}

According to theory, a sound innovation ecosystem should include the following components. First, a broad awareness and culture of innovation in the society, strict property rights and an intellectual property protection system, transparent business rules, fair market order and a complete mechanism for talent flow. Second, it must provide resources for education, scientific research, talent and capital to meet the needs of innovative companies. Third, it must produce a mechanism that promotes effective and integrative development of policies, production, learning and research, as well as advancing the role of the government and the market in resource allocation, accelerating industrialisation of scientific and technological achievements and maximising the realisation of economic and social benefits. Fourth, the government must pay close attention to innovation in economic development and, according to the needs at different stages of innovation and development, carry out policymaking and strategic planning to effectively promote innovation.

\section{Theory of innovation competency}

The innovation competency of companies is their ability to achieve innovation, improvement and advancement in all aspects of production and operation. It comprises the following six competencies:

1. Innovation input competency, which refers to the quantity and quality of the resources invested in a company's innovation activities, which is, in turn, the basis of that company's innovation activities. According to the internationally accepted method, company innovation input is divided into research and development $(\mathrm{R} \& \mathrm{D})$ input and non-R\&D input.

2. Innovation of R\&D competency, which is a key factor influencing a company's innovation, including its competency of $\mathrm{R} \& \mathrm{D}$ in terms of creating new technology and new products. 
3. Market innovation competency - that is, any innovative activity must ultimately be recognised by the market and be commercialised, so that all innovation input can be transformed into real economic and social benefits. This includes the competence to marketise new products and to adopt new technologies to transform existing markets.

4. Organisational innovation competency, which mainly includes the innovation competencies of the organisational system and function, the organisational management system and its rules and regulations.

5. Innovation of motivation competency, which helps stimulate a company's desire for innovation and encourages it to make this a reality. This competency can encourage employees to take the initiative and be forward-looking in their approach to innovation.

6. Innovation output competency, which refers to the capability to reduce costs, create markets and generate revenue through technological innovation, demonstrating the economic benefits and technological advances brought by such innovation.

\section{Two basic forms of innovation}

The term 'technological innovation' in this chapter refers to innovative activities in which new technologies are researched and developed; these technologies are directly utilised to establish new processes and produce new products. The term 'model innovation' refers to the innovative activities in which new models of production (excluding the establishment of new processes), organisational models and business models are researched and developed. Based on these definitions and the comparison of various international methods of innovation classification, we believe that almost all forms of innovation can be categorised as one of three types: 1) technological innovation, 2) model innovation or 3) hybrid innovation, which combines the first two types of innovation.

\section{Construction of an evaluation index for innovation competency of listed companies in China}

Drawing on the above theories and the research findings of similar projects in China, this chapter is a response to the need to promote the innovation and development of Chinese listed companies. Moreover, this chapter establishes, with the support of big data technology, the first evaluation index for Chinese listed companies' technological and model innovation. The research data mainly comes from three sources: the Wind Database (innovation inputs and innovation outputs), the Guotaian Database (technological innovation patent) and data mining from annual reports and announcements of listed companies (innovation environment, technological innovation, model innovation). 


\section{Composition of the index}

The innovation index system of Chinese listed companies consists of five major sectors: the innovation environment, innovation inputs, technological innovation, model innovation and innovation outputs. It should be noted that in this framework, technological innovation and model innovation are regarded as the intermediate processes between innovation input and innovation output.

Table 8.1 Index system of technological and model innovation competency of listed companies

\begin{tabular}{|l|l|}
\hline Primary indicator & Secondary indicator \\
\hline Innovation environment & Industry-university-research innovation alliance \\
\cline { 2 - 2 } & Innovative culture \\
\cline { 2 - 2 } & Innovation incentive mechanism \\
\hline \multirow{4}{*}{ Innovation inputs } & R\&D investment quantity and intensity \\
\cline { 2 - 2 } & Number and proportion of R\&D personnel \\
\cline { 2 - 2 } & Government funding for innovation \\
\hline Technological innovation & Number of patents (including application and authorisation) \\
\cline { 2 - 2 } & Technological innovation awards \\
\cline { 2 - 2 } & New product quantity and proportion \\
\hline Model innovation & Innovative organisation \\
\cline { 2 - 2 } & New marketing and new business \\
\hline \multirow{3}{*}{ Innovation outputs } & Full labour productivity \\
\cline { 2 - 2 } & Company value-benefit ratio \\
\cline { 2 - 2 } & Operating profit margin \\
\hline
\end{tabular}

\section{Index synthesis methods}

The system consists of five primary indicators: innovation environment, innovation inputs, technological innovation, model innovation and innovation outputs (Table 8.1). First, all secondary indicators of one primary indicator are nondimensionalised. The value of the secondary indicators and their associated weights are calculated according to the weighted average formula to obtain a score for the primary indicator. The score for each primary indicator is then weighted and averaged again to obtain an overall score for the index. 


\section{Evaluation of innovation competency of listed companies in China}

Among industries in China, the most active and competitive companies are domestic listed companies. Therefore, evaluation of the innovation competency of listed companies in China can provide a good picture of the structural distribution and overall trend of China’s innovation competency.

\section{Overall analysis of innovation competency}

\section{Innovation competency of listed companies is generally low}

China's securities market has long been oriented towards finance, and there is no effective incentive mechanism for company innovation and development. In addition, the lag in the reform of the securities market system has seen many internet innovation companies, represented by 'BAT', ${ }^{2}$ target overseas markets. The innovation competency of listed companies in China is generally weak and the total innovation competency score in the index is currently 43.94 (Table 8.2).

\section{Most listed companies in China are in the preliminary stage of innovation and development}

The innovation and development of listed companies in China reveal a development trend of 'relatively high input and relatively low output'.

Table 8.2 Innovation competency index of listed companies in China, 2017

\begin{tabular}{|l|l|l|l|l|l|l|}
\hline \multicolumn{2}{|c|}{ Overall indicator } & \multicolumn{5}{c|}{ Primary indicator } \\
\hline $\begin{array}{l}\text { Innovation } \\
\text { index }\end{array}$ & $\begin{array}{l}\text { Innovation } \\
\text { competency }\end{array}$ & $\begin{array}{l}\text { Innovation } \\
\text { environment }\end{array}$ & $\begin{array}{l}\text { Innovation } \\
\text { input }\end{array}$ & $\begin{array}{l}\text { Technological } \\
\text { innovation }\end{array}$ & $\begin{array}{l}\text { Model } \\
\text { innovation }\end{array}$ & $\begin{array}{l}\text { Innovation } \\
\text { output }\end{array}$ \\
\hline Overall & 43.94 & 53.42 & 45.8 & 40.77 & 39.09 & 40.61 \\
\hline
\end{tabular}

The scores for the technological innovation index, model innovation index and innovation output index of listed companies in China are significantly smaller than that of the innovation input index, which has a twofold importance: first, most of the listed companies in China are still in the preliminary stages of innovation, marked by high inputs, and only a few companies have entered the later innovation stage marked by high benefits. Second, there is a relatively low conversion efficiency of China's listed companies' innovation inputs. In the future, as China increases innovation inputs, it must also pay more attention to increasing the conversion efficiency of these inputs.

2 'BAT' is the acronym formed by the names of the three major Chinese internet companies: Baidu, Alibaba and Tencent. 


\section{Analysis of innovation competency according to region}

\section{Innovation activities of listed companies in China are highly concentrated in the east}

The regional distribution of China's top 500 companies in terms of innovation competency shows there are more innovative companies in the east and fewer in the west of the country. Among the top 500 companies, more than 80 per cent are in the east. The numbers of listed companies in the central, western and northeastern regions account for 11.4 per cent, 6.2 per cent and 2 per cent, respectively, of the total (see Figure 8.1).

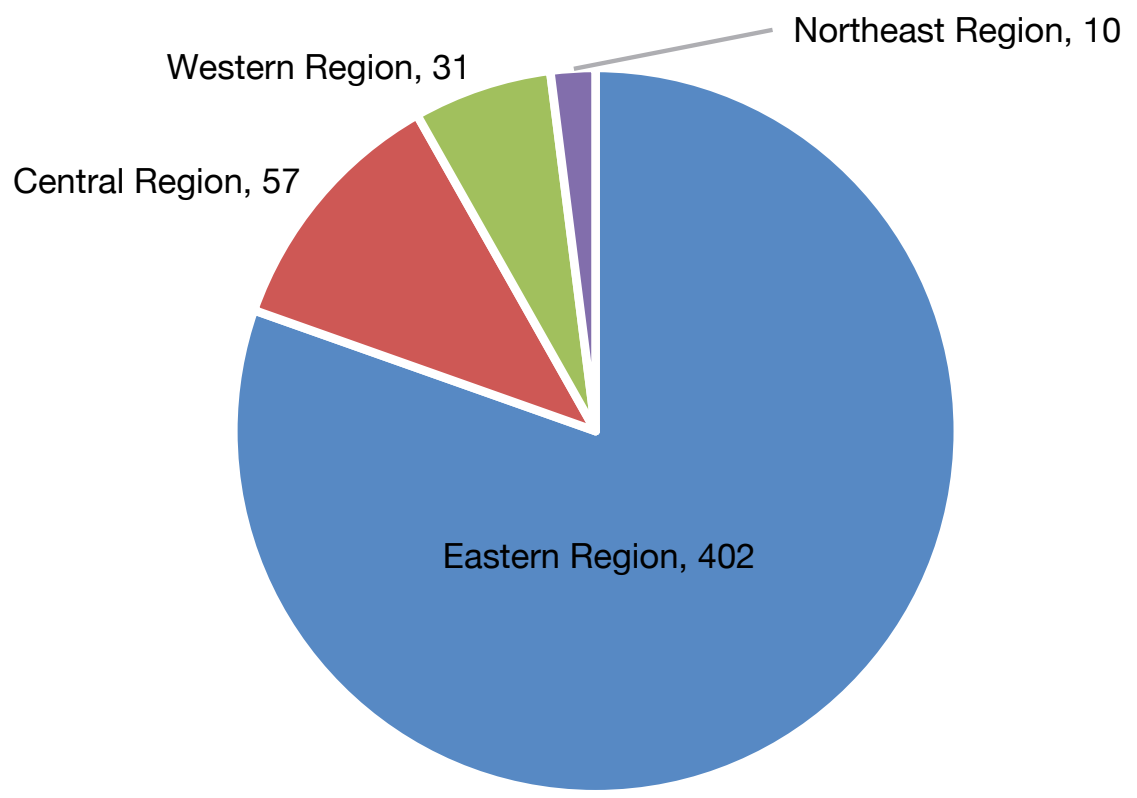

Figure 8.1 Distribution of top 500 listed enterprises for innovation capability in China, 2017

Source: Authors' calculation. 


\section{Spatial distribution of innovation competency by municipality and province takes a gradient descent}

The provinces and municipalities with a total innovation competency score of 63 or above are identified as the 'first echelon' (Table 8.3). Those in the first echelon have strong innovation competency and include Beijing, Guangdong Province, Zhejiang Province, Shanghai, Jiangsu Province, Fujian Province and Shandong Province.

The provinces and municipalities with a total innovation competency score of 53-63 form the 'second echelon', and their innovation competency is relatively strong. The second echelon includes 14 provinces and municipalities: Sichuan Province, Hubei Province, Anhui Province, Henan Province, Hunan Province, Liaoning Province, Tianjin, Hebei Province, Chongqing, Shaanxi Province, Jiangxi Province, Guizhou Province, Jilin Province and Yunnan Province.

The provinces and municipalities with a total innovation competency score of 53 or less are identified as the 'third echelon', and their innovation competency is relatively weak. The third echelon includes 10 provinces and municipalities: Xinjiang Uygur Autonomous Region, Guangxi Zhuang Autonomous Region, Heilongjiang Province, Gansu Province, Shanxi Province, Hainan Province, Inner Mongolia Autonomous Region, Tibet Autonomous Region, Qinghai Province and Ningxia Hui Autonomous Region.

The gradient distribution makes it obvious that innovation competency follows a descending pattern from eastern China to the central and then the western regions. All provinces and municipalities in the first echelon are in eastern China. The majority of places in the second echelon are in the central region, while in the third echelon-except for Hainan Province and Heilongjiang Province-all provinces and municipalities are in the west. 


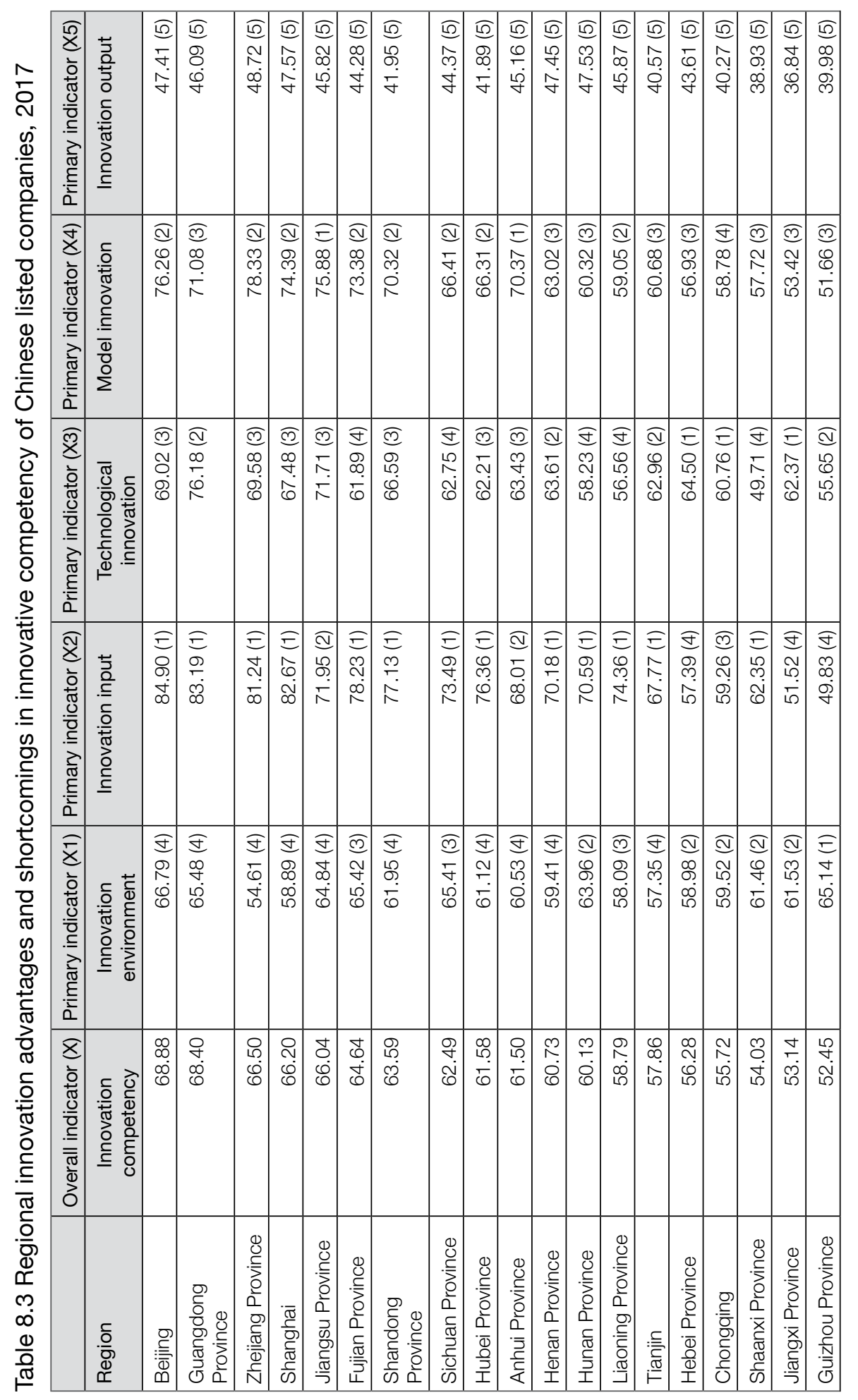


8. Innovation of Chinese listed enterprises

\begin{tabular}{|c|c|c|c|c|c|c|c|c|c|c|c|c|c|}
\hline 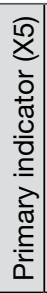 & 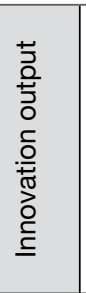 & 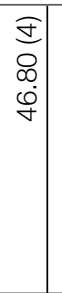 & 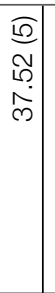 & $\begin{array}{l}\frac{\sigma}{6} \\
\overline{6} \\
\dot{q}\end{array}$ & 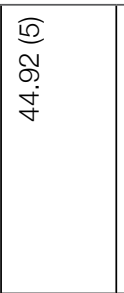 & $\begin{array}{l}\frac{\sigma}{\omega} \\
\frac{\sigma}{\sigma} \\
\stackrel{g}{q}\end{array}$ & \begin{tabular}{|l|}
$\underline{\omega}$ \\
0 \\
$N$ \\
$\infty$ \\
$\infty$
\end{tabular} & $\begin{array}{l}\sigma \\
0 \\
0 \\
0 \\
\omega \\
\infty\end{array}$ & 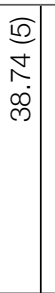 & 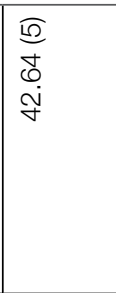 & $\begin{array}{l}\widehat{a} \\
0 \\
0 \\
\stackrel{0}{0}\end{array}$ & 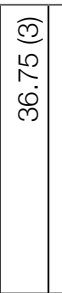 & 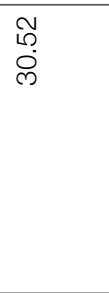 \\
\hline 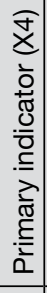 & 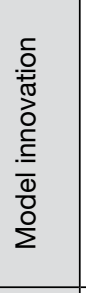 & 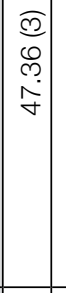 & 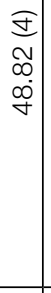 & \begin{tabular}{l}
$\widehat{\mathbb{d}}$ \\
\multirow{0}{0}{} \\
î
\end{tabular} & $\begin{array}{l}\mathcal{J} \\
\mathcal{J} \\
\stackrel{b}{f}\end{array}$ & 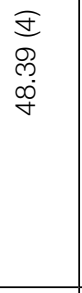 & $\begin{array}{l}\widehat{\widehat{c}} \\
\hat{0} \\
\bar{b}\end{array}$ & $\begin{array}{l}\vec{f} \\
\stackrel{2}{0} \\
\dot{f}\end{array}$ & $\begin{array}{l}\widehat{\underline{m}} \\
\stackrel{5}{p} \\
\vec{g}\end{array}$ & $\begin{array}{l}\widehat{\widehat{a}} \\
\bar{\alpha} \\
\dot{q} \\
\dot{q}\end{array}$ & $\begin{array}{l}\frac{\sigma}{\sigma} \\
\stackrel{\sigma}{C} \\
\stackrel{\infty}{\infty}\end{array}$ & $\begin{array}{l}0 \\
0 \\
\delta \\
o \\
\dot{\rho} \\
0\end{array}$ & $\underset{\substack{\sim \\
\stackrel{\sim}{\infty}}}{\stackrel{\sim}{\infty}}$ \\
\hline 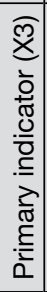 & 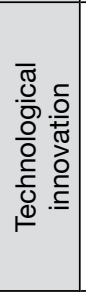 & $\begin{array}{c}\frac{\sigma}{b} \\
\stackrel{v}{0} \\
\frac{b}{b} \\
\end{array}$ & $\begin{array}{l}\overline{0} \\
\bar{c} \\
\dot{b} \\
b\end{array}$ & 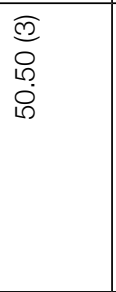 & 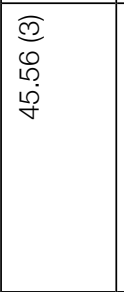 & $\begin{array}{l}\widehat{\widehat{c}} \\
\text { g } \\
\stackrel{\sigma}{f}\end{array}$ & $\begin{array}{l}\widehat{a} \\
\vec{d} \\
\tilde{v} \\
i\end{array}$ & $\begin{array}{l}\widehat{\mathbb{N}} \\
0 \\
\hat{ల} \\
\hat{0}\end{array}$ & 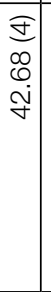 & $\begin{array}{l}\widehat{\widehat{m}} \\
\hat{\circ} \\
\hat{\phi}\end{array}$ & $\begin{array}{l}\mathbb{J} \\
\mathbb{E} \\
\stackrel{\infty}{\infty} \\
0\end{array}$ & $\left|\begin{array}{c}\widehat{\widehat{N}} \\
\widehat{N} \\
\hat{m}\end{array}\right|$ & 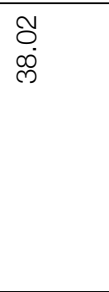 \\
\hline 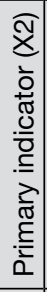 & 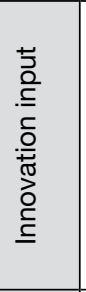 & 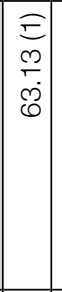 & 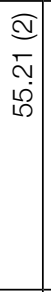 & 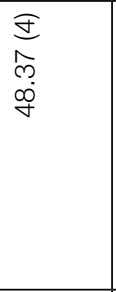 & $\begin{array}{l}\widehat{\mathbb{d}} \\
\mathbf{0} \\
0 \\
0 \\
0\end{array}$ & $\begin{array}{l}E \\
\bar{E} \\
\bar{N} \\
\dot{b}\end{array}$ & 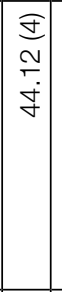 & $\begin{array}{l}\widehat{m} \\
\bar{\sigma} \\
\dot{\sigma}\end{array}$ & 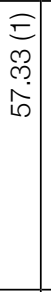 & 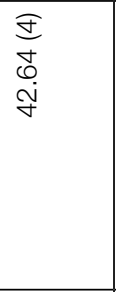 & 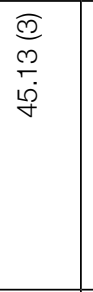 & $\left|\begin{array}{c}\widehat{\omega} \\
\stackrel{N}{N} \\
\dot{\omega}\end{array}\right|$ & $\begin{array}{l}\stackrel{\infty}{\infty} \\
\text { m. } \\
\stackrel{m}{0}\end{array}$ \\
\hline 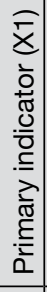 & 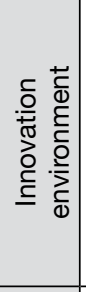 & \begin{tabular}{|c|}
$\widehat{\widehat{a}}$ \\
0 \\
0 \\
$\infty$ \\
$\infty$ \\
\\
\end{tabular} & 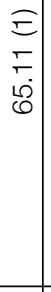 & $\begin{array}{l}\text { E् } \\
\stackrel{0}{0} \\
0 \\
\infty \\
0\end{array}$ & \begin{tabular}{l}
$\bar{E}$ \\
o \\
\multirow{L}{*}{}
\end{tabular} & $\begin{array}{l}\widehat{\mathbb{d}} \\
\overline{0} \\
\bar{L}\end{array}$ & $\begin{array}{l}E \\
D \\
D \\
\dot{D}\end{array}$ & $\begin{array}{l}2 \\
0 \\
b \\
i \\
i\end{array}$ & \begin{tabular}{l}
$\widehat{a}$ \\
\multicolumn{1}{c}{} \\
$\dot{b}$
\end{tabular} & 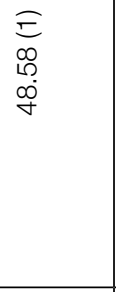 & $\begin{array}{l}\text { E } \\
\mathbb{0} \\
0 \\
\dot{B}\end{array}$ & 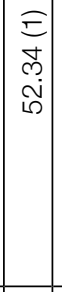 & 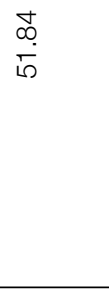 \\
\hline $\begin{array}{l}\bar{x} \\
\overline{0} \\
\overline{0} \\
\frac{0}{0} \\
. \\
\overline{0} \\
\overline{0} \\
\overline{0} \\
0\end{array}$ & 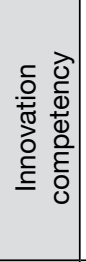 & 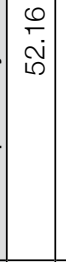 & 怘 & $\begin{array}{l}\mathscr{8} \\
\dot{\sigma}\end{array}$ & $\begin{array}{l}\infty \\
\infty \\
\infty \\
o j \\
q\end{array}$ & $\begin{array}{l}\hat{\lambda} \\
\stackrel{\alpha}{\alpha}\end{array}$ & 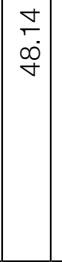 & $\begin{array}{l}\stackrel{\rho}{m} \\
\stackrel{\gamma}{\sigma}\end{array}$ & 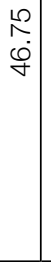 & $\begin{array}{l}\hat{0} \\
\dot{b} \\
\dot{g}\end{array}$ & $\begin{array}{l}\tilde{O} \\
\dot{J}\end{array}$ & $\begin{array}{l}\vec{J} \\
\dot{p} \\
\mid\end{array}$ & $\begin{array}{l}\tilde{\omega} \\
\infty \\
\infty \\
\infty\end{array}$ \\
\hline & 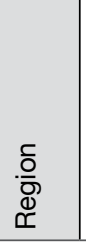 & 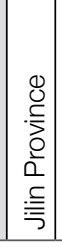 & 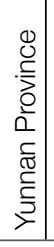 & 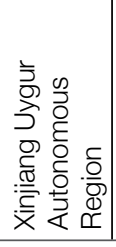 & 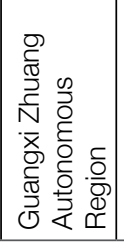 & 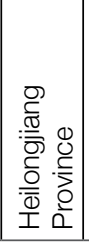 & 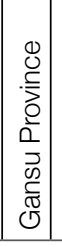 & 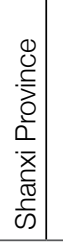 & 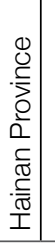 & 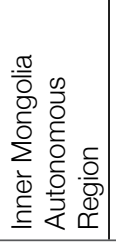 & 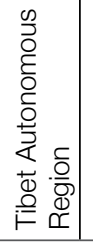 & 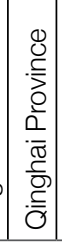 & 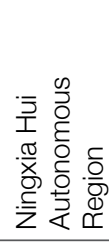 \\
\hline
\end{tabular}




\section{Analysis of innovation competency according to industry}

\section{High-tech manufacturing industries and modern service industries have strong innovation competency, and traditional industries have relatively weaker innovation competency}

According to the comparative analysis of the overall index of innovation competency, industries with a higher total innovation competency index are mainly high-tech manufacturing and modern service industries (Table 8.4). Industries with lower innovation competency are mainly traditional manufacturing and traditional service industries. The innovation competency scores of high-tech manufacturing and modern service industries are 48.52 and 48.56 , respectively.

Innovation competency scores for traditional manufacturing and service industries are 41.96 and 37.07, respectively, which are 6.56 and 11.49 points less than the high-tech manufacturing and modern service industries, respectively.

\section{Service industries focus on model innovation and manufacturing industries focus on technological innovation}

Innovation in service industries is dominated by model innovation. The score for the model innovation index of service industries is 3.74 points higher than the technological innovation score. The scores for model innovation for information transfer, software and information technology services, culture, sports and entertainment, leasing and commercial services, finance and board and lodging services are 6 points higher than the scores for the technological innovation index.

Innovation in manufacturing industries is dominated by technological innovation. The technological innovation index score for manufacturing is 5.17 points higher than the model innovation score. The technological innovation index scores for the comprehensive utilisation of waste resources, processing of timber, manufacturing of wood, bamboo, rattan, palm and straw products, smelting, rolling and pressing of ferrous metals and smelting, rolling and pressing of nonferrous metals are 10 points higher than their model innovation scores. 
8. Innovation of Chinese listed enterprises

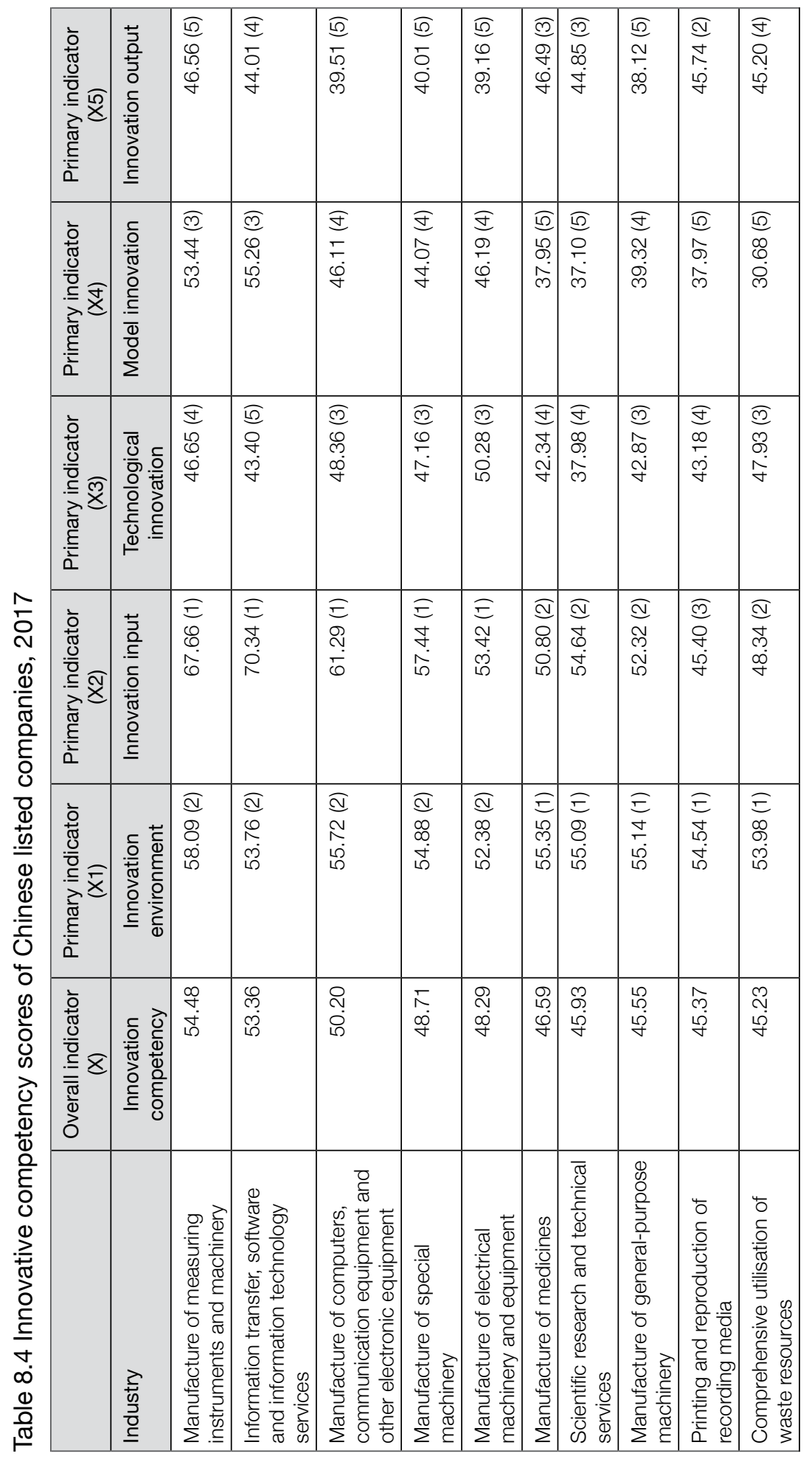




\begin{tabular}{|c|c|c|c|c|c|c|c|c|c|c|c|c|c|c|c|}
\hline 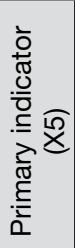 & 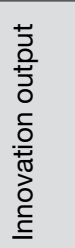 & $\begin{array}{l}\widehat{\widehat{g}} \\
\stackrel{0}{0} \\
\underline{\sigma} \\
\dot{\sigma}\end{array}$ & 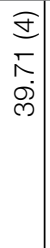 & 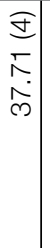 & 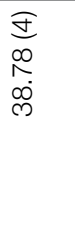 & $\begin{array}{l}\mathcal{J} \\
\stackrel{1}{ } \\
N \\
\infty \\
\infty\end{array}$ & $\begin{array}{l}\overparen{D} \\
m \\
0 \\
00 \\
\infty\end{array}$ & 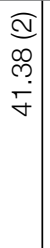 & 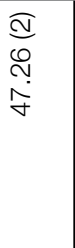 & $\begin{array}{l}\vec{J} \\
\sigma \\
\bar{\sigma} \\
\dot{m}\end{array}$ & 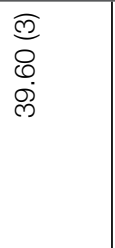 & 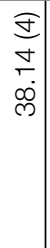 & $\begin{array}{l}\widehat{0} \\
\dot{b} \\
0 \\
\dot{0} \\
0\end{array}$ & $\begin{array}{l}\widehat{\sigma} \\
\stackrel{+}{0} \\
\dot{+} \\
\dot{+}\end{array}$ & $\begin{array}{l}\widetilde{I} \\
\widetilde{N} \\
\infty \\
\dot{J}\end{array}$ \\
\hline 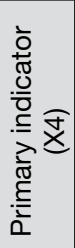 & 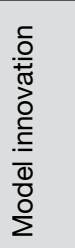 & $\begin{array}{l}\widetilde{0} \\
\infty \\
0 \\
\dot{j} \\
\infty\end{array}$ & $\begin{array}{l}\widetilde{0} \\
\sigma \\
\infty \\
\infty\end{array}$ & 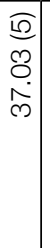 & $\begin{array}{l}\widehat{a} \\
\hat{N} \\
\infty \\
\infty \\
\infty\end{array}$ & 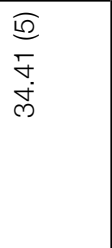 & 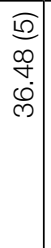 & 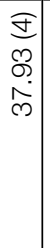 & 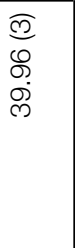 & $\begin{array}{l}\mathbb{b} \\
\stackrel{2}{N} \\
0 \\
\infty\end{array}$ & $\begin{array}{l}\frac{\widehat{D}}{\infty} \\
N \\
\stackrel{\infty}{\infty}\end{array}$ & $\begin{array}{l}\widetilde{0} \\
m \\
\infty \\
\dot{\infty} \\
\dot{\infty}\end{array}$ & 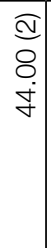 & 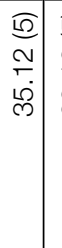 & $\begin{array}{l}\frac{\sigma}{0} \\
\frac{0}{\infty} \\
\infty \\
\infty\end{array}$ \\
\hline 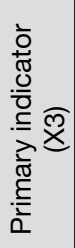 & 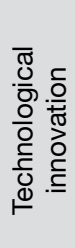 & $\begin{array}{l}\text { త্ } \\
\stackrel{N}{N} \\
\infty \\
\infty\end{array}$ & $\begin{array}{l}\widehat{\mathbb{N}} \\
\hat{\varphi} \\
\dot{P} \\
\forall\end{array}$ & 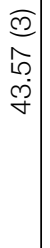 & 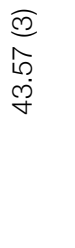 & $\begin{array}{l}\text { लु } \\
\infty \\
\stackrel{\infty}{ } \\
\stackrel{f}{f}\end{array}$ & $\begin{array}{l}\text { लु } \\
\stackrel{1}{0} \\
\dot{y}\end{array}$ & 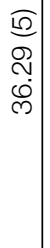 & 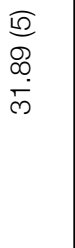 & 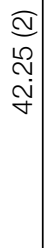 & $\begin{array}{l}\text { J } \\
0 \\
0 \\
0 \\
\infty\end{array}$ & $\begin{array}{c}\widetilde{\sigma} \\
0 \\
\dot{\sigma} \\
\dot{\sigma}\end{array}$ & $\begin{array}{l}\widetilde{0} \\
\underset{N}{N} \\
\dot{q}\end{array}$ & 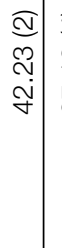 & 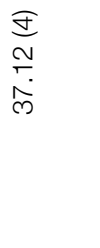 \\
\hline 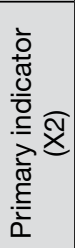 & 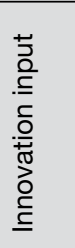 & $\begin{array}{l}\text { త్ } \\
\text { ஓ } \\
\text { g. }\end{array}$ & 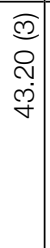 & $\begin{array}{l}\text { త্ } \\
\bar{\sigma} \\
\dot{\sigma}\end{array}$ & $\begin{array}{l}\text { త্ } \\
\dot{\infty} \\
\dot{\sigma} \\
\dot{\sigma}\end{array}$ & $\begin{array}{l}\text { త్ } \\
\text { Jै } \\
\text { Nิ }\end{array}$ & $\begin{array}{l}\mathbb{a} \\
\dot{J} \\
\dot{g}\end{array}$ & 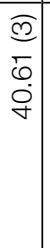 & 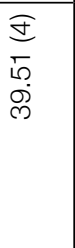 & 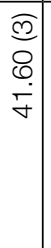 & $\begin{array}{l}\frac{\widehat{d}}{N} \\
\frac{\mathfrak{g}}{\dot{\sigma}}\end{array}$ & 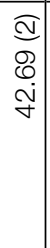 & $\begin{array}{l}\overparen{J} \\
0 \\
\tilde{-} \\
\tilde{m}\end{array}$ & 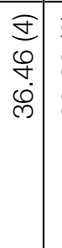 & $\begin{array}{l}\widehat{0} \\
\infty \\
0 \\
\infty \\
\infty\end{array}$ \\
\hline 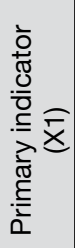 & 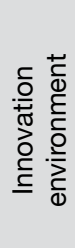 & $\begin{array}{l}\underset{E}{N} \\
\underset{N}{0} \\
\infty \\
\infty\end{array}$ & $\begin{array}{l}E \\
0 \\
0 \\
0 \\
0\end{array}$ & $\begin{array}{l}\text { E् } \\
0 \\
10 \\
N \\
0 \\
0\end{array}$ & $\begin{array}{l}\text { E } \\
\mathscr{Q} \\
\text { ले } \\
10\end{array}$ & 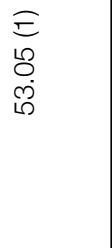 & 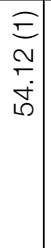 & $\begin{array}{l}E \\
\qquad 8 \\
0 \\
\varnothing \\
0\end{array}$ & $\begin{array}{l}\text { E } \\
\dot{0} \\
0 \\
\infty \\
\circ\end{array}$ & $\begin{array}{l}E \\
\infty \\
\infty \\
\tilde{D}\end{array}$ & $\begin{array}{l}\text { E } \\
\text { Dे } \\
\stackrel{5}{5}\end{array}$ & $\begin{array}{l}E \\
\underline{E} \\
\bar{N} \\
\tilde{D}\end{array}$ & $\begin{array}{l}\widetilde{E} \\
\bar{\tau} \\
\dot{v} \\
\tilde{\sigma}\end{array}$ & $\begin{array}{l}0 \\
E \\
0 \\
1 \\
0\end{array}$ & $\begin{array}{l}\text { E } \\
8 \\
\varnothing \\
\ddot{1}\end{array}$ \\
\hline \multirow[t]{2}{*}{ 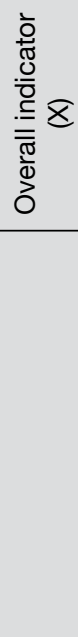 } & 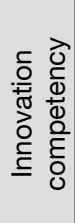 & 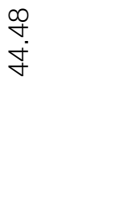 & $\begin{array}{l}\stackrel{L}{f} \\
\dot{\nabla}\end{array}$ & $\begin{array}{l}\stackrel{⿱}{0} \\
\stackrel{f}{*}\end{array}$ & $\begin{array}{l}\stackrel{\infty}{N} \\
\stackrel{v}{\forall}\end{array}$ & $\begin{array}{l}8 \\
\circ \\
\dot{\forall}\end{array}$ & $\begin{array}{l}\bar{\infty} \\
\dot{\sigma} \\
\dot{\sigma}\end{array}$ & $\begin{array}{l}\hat{r} \\
\stackrel{\mathscr{g}}{\gamma}\end{array}$ & $\begin{array}{l}\bar{\Omega} \\
\stackrel{\gamma}{\forall}\end{array}$ & 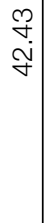 & $\begin{array}{l}\hat{N} \\
\underset{\gamma}{\forall}\end{array}$ & 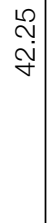 & $\begin{array}{l}\stackrel{\circ}{0} \\
\stackrel{\sim}{f}\end{array}$ & $\begin{array}{l}\infty \\
\infty \\
\dot{\nabla} \\
\dot{\gamma}\end{array}$ & $\begin{array}{l}\mathscr{Q} \\
\stackrel{\wp}{\sigma}\end{array}$ \\
\hline & 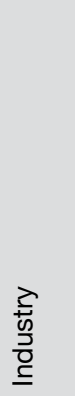 & 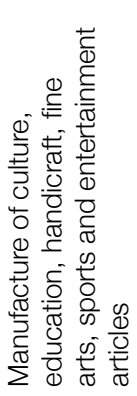 & 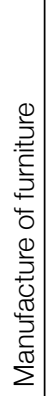 & 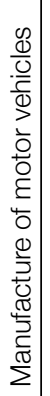 & 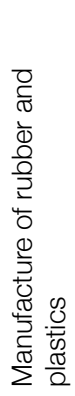 & 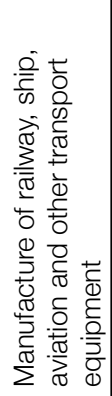 & 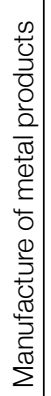 & 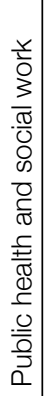 & 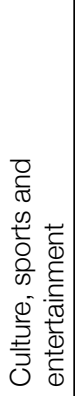 & 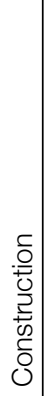 & 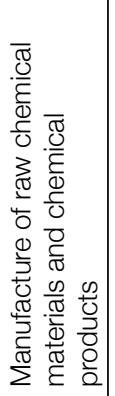 & 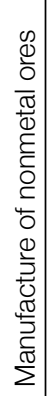 & 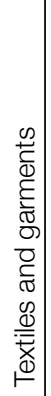 & \begin{tabular}{l|l} 
& \\
& \\
0 \\
8 \\
0 \\
$\frac{0}{0}$ \\
0 \\
0 \\
0 \\
2 \\
0 \\
$\frac{\pi}{5}$ \\
2 \\
$\frac{1}{10}$ \\
2
\end{tabular} & 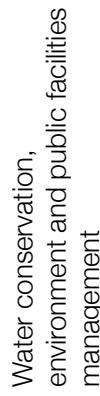 \\
\hline
\end{tabular}




\begin{tabular}{|c|c|c|c|c|c|c|c|c|c|c|c|c|c|c|c|}
\hline 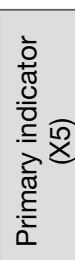 & 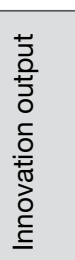 & $\begin{array}{l}\text { త్ } \\
\text { Oु } \\
\dot{f}\end{array}$ & 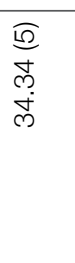 & 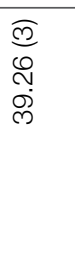 & 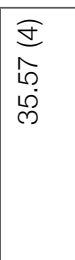 & $\begin{array}{l}\widehat{\widetilde{U}} \\
\emptyset \\
\emptyset \\
\dot{\nabla}\end{array}$ & $\begin{array}{l}\sigma \underline{\sigma} \\
\overline{0} \\
\dot{m}\end{array}$ & $\begin{array}{l}\widehat{\mathbb{V}} \\
\stackrel{O}{N} \\
\stackrel{y}{\forall}\end{array}$ & 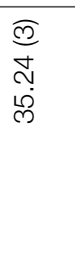 & $\begin{array}{l}\widehat{\infty} \\
\underset{N}{N} \\
\stackrel{\infty}{\infty}\end{array}$ & $\begin{array}{l}\widehat{D} \\
10 \\
0 \\
\stackrel{D}{N}\end{array}$ & $\begin{array}{l}\widehat{ত} \\
8 \\
\dot{\square} \\
\dot{y}\end{array}$ & $\begin{array}{l}\overparen{ \pm} \\
\sigma \\
\dot{\rho} \\
\text { | }\end{array}$ & $\begin{array}{l}\widehat{E} \\
\omega \\
\Omega \\
\dot{1} \\
\llcorner\end{array}$ & $\begin{array}{l}\widehat{\widetilde{v}} \\
\tilde{m} \\
\dot{\sigma} \\
\dot{\infty}\end{array}$ \\
\hline 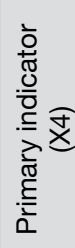 & 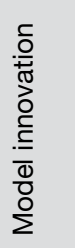 & $\begin{array}{l}\text { लु } \\
\infty \\
\text { N } \\
\stackrel{f}{q}\end{array}$ & 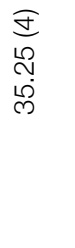 & $\begin{array}{l}\overparen{D} \\
\stackrel{5}{N} \\
\stackrel{D}{D}\end{array}$ & 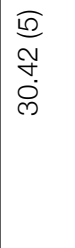 & 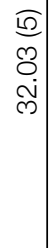 & $\begin{array}{l}\mathcal{J} \\
\stackrel{5}{0} \\
N \\
0 \\
0\end{array}$ & $\begin{array}{l}\overparen{J} \\
0 \\
\sim \\
\dot{\infty}\end{array}$ & $\begin{array}{l}\mathcal{\Xi} \\
\infty \\
0 \\
\dot{\rho} \\
\stackrel{\rho}{ }\end{array}$ & 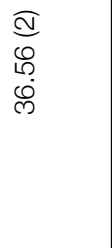 & 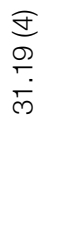 & $\begin{array}{l}\widetilde{E} \\
8 \\
0 \\
\tilde{\infty}\end{array}$ & 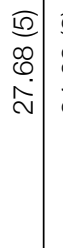 & $\begin{array}{l}\text { लू } \\
\infty \\
0 \\
\dot{ल}\end{array}$ & 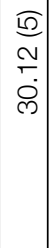 \\
\hline 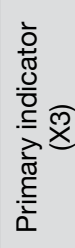 & 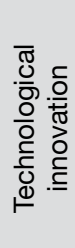 & $\begin{array}{l}\text { E్ } \\
\bar{\sigma} \\
\infty \\
\ddot{\infty}\end{array}$ & 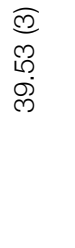 & $\begin{array}{l}\text { E } \\
\infty \\
\infty \\
\infty \\
\dot{q}\end{array}$ & 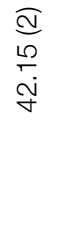 & 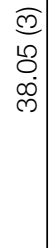 & 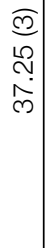 & 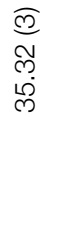 & $\begin{array}{l}\text { త్ } \\
i 0 \\
\infty \\
\dot{0} \\
0\end{array}$ & $\begin{array}{l}\mathcal{\Xi} \\
\stackrel{N}{\wedge} \\
\dot{B}\end{array}$ & 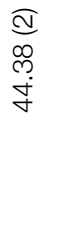 & 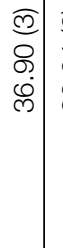 & 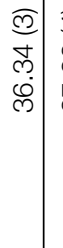 & 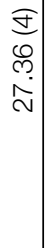 & 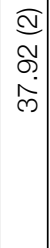 \\
\hline 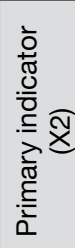 & 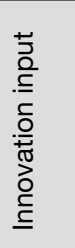 & $\begin{array}{l}\text { ச } \\
\text { ه } \\
\ddot{ల}\end{array}$ & 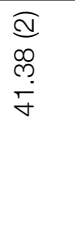 & $\begin{array}{l}\text { Ð } \\
\text { ठ } \\
\text { ले }\end{array}$ & $\begin{array}{l}\widehat{\widehat{D}} \\
\stackrel{N}{N} \\
\dot{\sigma}\end{array}$ & 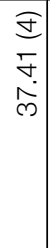 & $\begin{array}{l}\text { త్ } \\
\dot{m} \\
\stackrel{m}{ }\end{array}$ & $\begin{array}{l}\frac{\sigma}{0} \\
\frac{1}{n} \\
0 \\
\dot{\infty}\end{array}$ & 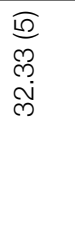 & $\begin{array}{l}\sqrt{0} \\
\infty \\
1 \\
\infty \\
\bar{\infty}\end{array}$ & $\begin{array}{l}\text { लु } \\
\infty \\
0 \\
\stackrel{\rho}{\infty}\end{array}$ & $\begin{array}{l}\mathcal{\Xi} \\
\check{N} \\
\ddot{\infty}\end{array}$ & 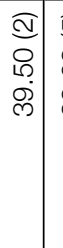 & 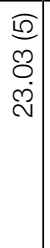 & 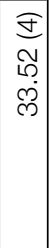 \\
\hline 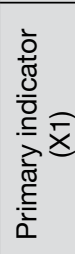 & 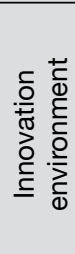 & 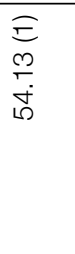 & 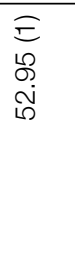 & $\begin{array}{l}\widehat{\mathcal{N}} \\
\mathbb{M} \\
\stackrel{f}{\forall}\end{array}$ & 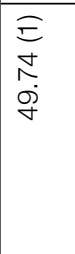 & $\begin{array}{l}\hat{E} \\
\dot{J} \\
\dot{g} \\
\dot{q}\end{array}$ & $\begin{array}{l}E \\
\hat{E} \\
\vdots \\
0 \\
0\end{array}$ & $\begin{array}{l}\widehat{E} \\
\infty \\
\infty \\
\infty \\
0 \\
0\end{array}$ & 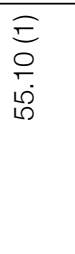 & $\begin{array}{l}\text { E } \\
0 \\
N \\
0 \\
0 \\
0\end{array}$ & $\begin{array}{l}\widehat{E} \\
\hat{\infty} \\
\infty \\
\text { g }\end{array}$ & 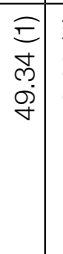 & $\begin{array}{l}E \\
0 \\
0 \\
0 \\
\tilde{N}\end{array}$ & $\begin{array}{c}\widetilde{\mathfrak{U}} \\
\infty \\
\overline{\tilde{N}} \\
\tilde{\omega}\end{array}$ & $\begin{array}{l}\widehat{E} \\
\bar{\sigma} \\
\dot{0} \\
\llcorner\end{array}$ \\
\hline 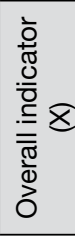 & 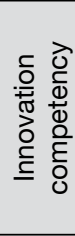 & 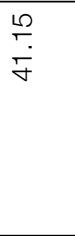 & $\begin{array}{l}\text { o } \\
0 \\
\text { वे }\end{array}$ & $\begin{array}{l}\widetilde{\gamma} \\
\text { ơ }\end{array}$ & $\begin{array}{l}\text { ळे } \\
\text { ळे }\end{array}$ & 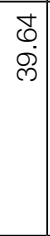 & 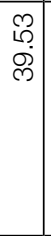 & $\begin{array}{l}\text { ले } \\
\text { ले } \\
\text { ल) }\end{array}$ & $\begin{array}{l}\text { Oे } \\
\infty \\
\infty \\
\varnothing\end{array}$ & $\begin{array}{l}\infty \\
\infty \\
\infty \\
\infty \\
\infty\end{array}$ & $\begin{array}{l}\infty \\
\infty \\
\infty \\
\infty \\
\infty\end{array}$ & $\begin{array}{l}0 \\
0 \\
\infty \\
\infty\end{array}$ & $\begin{array}{l}o \\
+ \\
\infty \\
0\end{array}$ & $\begin{array}{l}\text { ले } \\
\infty \\
\infty \\
\infty\end{array}$ & \begin{tabular}{|l}
$\infty$ \\
$\stackrel{\infty}{\rho}$ \\
$\mathcal{M}$ \\
$m$
\end{tabular} \\
\hline & 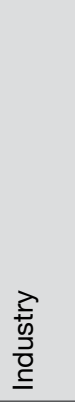 & 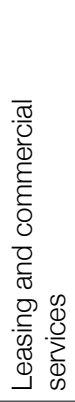 & 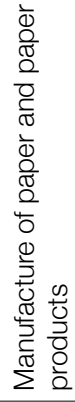 & 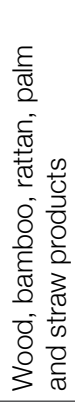 & 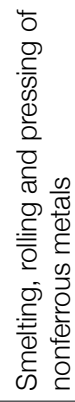 & 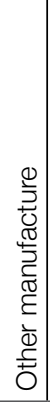 & 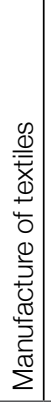 & 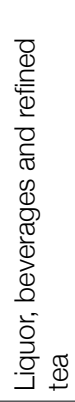 & 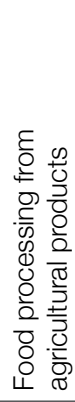 & 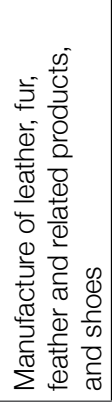 & 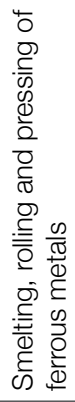 & $\begin{array}{l} \\
\\
0 \\
\frac{1}{0} \\
\frac{D}{0} \\
\frac{0}{0} \\
\frac{0}{0} \\
\frac{0}{0} \\
0\end{array}$ & 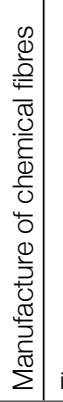 & 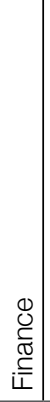 & 异 \\
\hline
\end{tabular}


The Chinese Economic Transformation

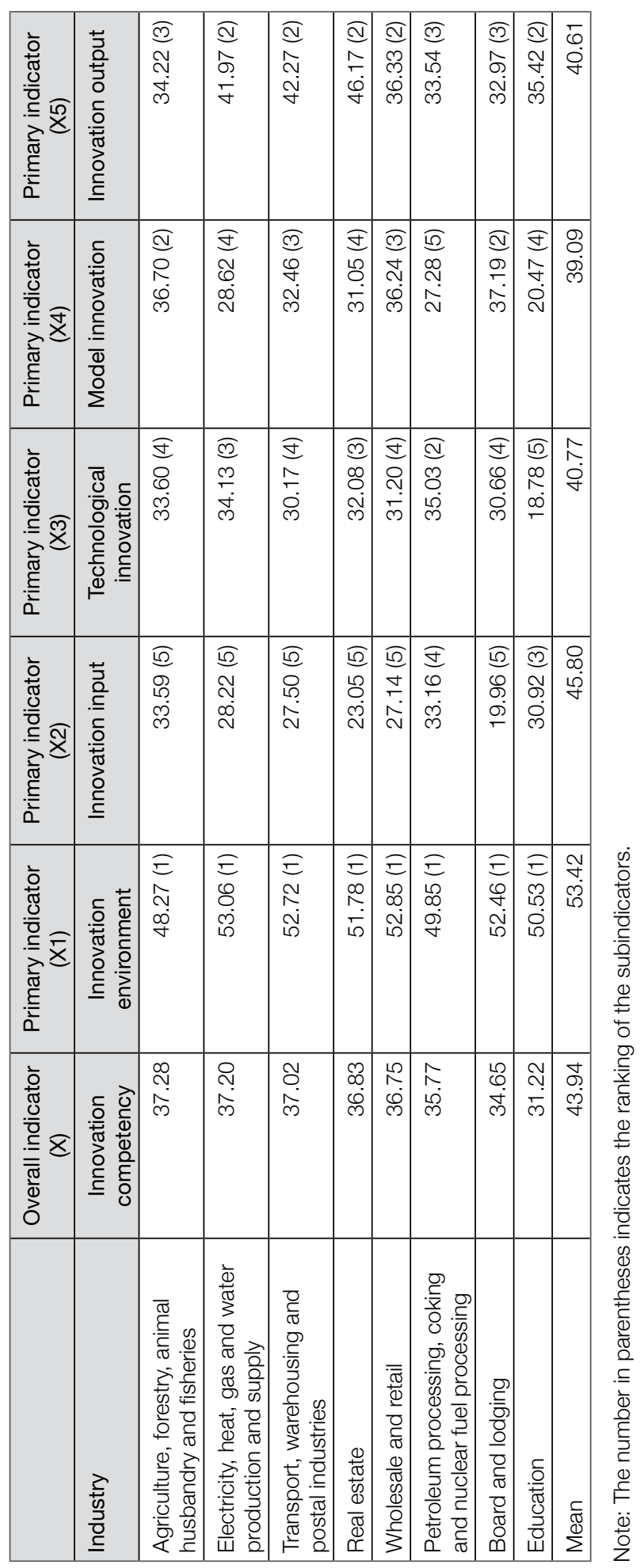




\section{Conclusions}

\section{Chinese company innovation is still in the early stage of development}

The process of company innovation and development consists of an early highinput stage and a late high-yield stage. At the end of 2017, China's strategy for innovation-driven development had been in operation for only five years, and the innovation and development of Chinese companies remain in the early stages. The innovation competency of listed companies is generally low, which is in line with the general trend of innovation and development. The evaluation results show that, with the implementation of the innovation-driven strategy, the continuous promotion of the government's reforms to delegate power, streamline administration and optimise government services and to promote large-scale entrepreneurship and innovation have led to significant improvements in the external environment for the innovation and development of Chinese companies. This has laid a robust foundation for the sustainable and sound development of company innovation.

It should be noted that the level of innovation and development of Chinese companies is due mainly to a small number of leading companies, while the innovation competency of most listed companies can, and should, be improved. A favourable pattern in which leading companies help smaller ones so as to achieve coordinated development has not yet been established.

\section{Distribution of China's listed companies according to innovation competency follows a gradient descent}

This study shows that, compared with the traditional factor-driven economy, an innovation-driven economy can lead to a concentration of resources. China's eastern region has the advantage of many resources-such as talent, capital, culture, internationalisation, reform and opening-up - and has become an area of concentration for Chinese company innovation and development. Among the top 500 innovative companies, those in the east account for 80 per cent of the total, with 44.6 per cent in Guangdong and Beijing alone. Guangdong and Beijing have assumed leading positions in company innovation and development on a national scale.

At the same time, the central region has become the main area for industrial transfer from the east and is strongly influenced by innovation activities in the eastern region. The central region has relatively good resources in terms of talent, capital and culture, and its innovation competency is ranked second in the country. In contrast, the mid-west and north-east regions have relatively few innovative resources, and therefore overall company innovation competency is weak. 


\section{Active innovation in high-tech manufacturing and modern service industries}

Company innovation activities are often carried out based on new technologies and models of operation. In recent years, to accelerate the upgrading and transformation of China's industrial structure, the central government has implemented a number of supporting policies that have effectively promoted the innovation and development of China's high-tech manufacturing industries and modern service industries. Among these industries, information transfer, software and information technology services, the manufacturing of computers, communication equipment and other electronic equipment, the manufacturing of electrical machinery and equipment, the manufacturing of special equipment and pharmaceutical manufacturing have become the five major innovative industries.

\section{Comments on innovation policies for firms in China}

In China, the rudiments of capitalism were already evident as long ago as the Song Dynasty. In the Ming Dynasty, there was a large-scale coal industry, which included exploration for and utilisation of natural gases, and a rather advanced iron and steel industry, which had already solved technical problems around the casting of iron and steel that Europeans were not able to figure out until the nineteenth century. However, the Industrial Revolution did not take place in China. The key reason was the longterm suppression of Chinese entrepreneurs' innovative spirit throughout history.

In September 2017, the State Council issued a policy document entitled 'Opinions Regarding Making a Healthy Growing Environment for Entrepreneurs, Spreading Excellent Entrepreneurial Spirit and Better Exercising Entrepreneurs' Roles'. The chairman of the board of Alibaba Group noted that this document marked 'a great leap forward in thinking since 2,000 years ago in China' (Sina Technology 2017). This document and the gradual introduction of relevant supporting policies will undoubtedly shape China's future development.

To encourage enterprise innovation, the Chinese Government has over the years issued hundreds of relevant policies, with the number reaching the tens of thousands when coupled with policies introduced by local governments. These policies can be grouped into the following categories:

1. Increasing efforts to protect intellectual property and establish and perfect the legal system governing intellectual property.

2. Promoting reforms to delegate power, streamline administration and optimise government services, and build a market environment in which entrepreneurs can compete fairly. Propelling the system of negative listing of market access 
and guaranteeing that all kinds of market subjects can legally and equally enter industries, fields and services outside the negative list. Opposing local protectionism, revising local regulations that hinder fair competition and establishing a unified market that allows the free flow of production factors.

3. Encouraging local governments to be bold and establishing a mechanism that tolerates mistakes and is adventurous.

4. Promoting close cooperation between universities, research institutes and enterprises, integrating three-party resources, improving innovation capacities and increasing the conversion efficiency of technological achievements.

5. Establishing and perfecting service platform systems that support entrepreneurial innovation. There are more than 5,500 maker spaces in China, along with more than 4,000 high-tech business incubators and over 970 innovation and startup platforms built by central government enterprises.

6. Some 120 innovation and startup model bases have been established. Through exploration and practise, successes can be promoted nationwide.

7. Boosting the construction of an innovation mechanism that involves cooperation between large, medium and small enterprises. Through an industry chain, these enterprises can co-establish innovation platforms, open innovation resources and engage in cooperative innovation activities.

8. Increasing efforts to support innovation-based enterprises in terms of finance and taxation policies and offering favourable fiscal terms to these enterprises.

9. Broadening the direct financing channels of innovation-based enterprises, and prioritising bond issues, financing and public offerings of medium and small tech enterprises.

As can be seen, to effectively promote the innovative development of enterprises, the Chinese Government has not only offered innovation-based enterprises taxation and financial support, but also introduced a series of supportive legal, social and administrative policies. These efforts have yielded remarkable results.

Based on various local surveys, there are two major flaws in the policies aimed at encouraging entrepreneurial innovation in China. The first is that some policies have not yet been fully formulated, such as the imperfect system of intellectual property protection. And the second is that some policies are not completely implemented due to various interruptions. These problems are bound to occur in the development process of a transitional country. Along with the continual and thorough implementation of innovation-driven strategies, these problems will gradually be effectively alleviated or resolved.

With the perfecting of the policy system that supports entrepreneurial innovation, Chinese enterprises will move from the early innovation stage of high investment to the late stage of high yields. This is the main reason the innovation of Chinese enterprises enjoys a rosy prospect. 


\section{References}

Breschi, S., Malerba, F. and Orsenigo, L. (2010), Technological regimes and Schumpeterian patterns of innovation, Economic Journal 110(463): 388-410. doi.org/10.1111/14680297.00530 .

Cao, H.J., Zhao, X. and Huang, S.J. (2009), Research on assessment systems of enterprises' independent innovation, [in Chinese], China Industrial Economies 9: 105-14.

Chen, K. and Guan, J. (2011), Mapping the functionality of China's regional innovation systems: A structural approach, China Economic Review 22(1): 10-27. doi.org/10.1016/ j.chieco.2010.08.002.

Chung, S. (2002), Building a national innovation system through regional innovation systems, Technovation 22(8): 485-91. doi.org/10.1016/S0166-4972(01)00035-9.

Cooke, P. (1997), Regional innovation systems: Institutional and organizational dimensions, Research Policy 26(4-5): 475-91. doi.org/10.1016/S0048-7333(97)00025-5.

Cooke, P. (2011), Transition regions: Regional-national eco-innovation systems and strategies, Progress in Planning 76(3): 105-46. doi.org/10.1016/j.progress.2011.08.002.

Dang, W.J., Zhang, Z.Y. and Kang, J.J. (2008), The impact of the regional innovation environment on regional innovation capability, [in Chinese], China Soft Science 3: 52-7.

Edwards, G. (2013), Regions and innovation: A reflection, in S. Kinnear, K. Charters and P. Vitartas (eds), Regional Advantage and Innovation: Achieving Australia's national outcomes, Heidelberg: Springer-Verlag.

Fagerberg, J.E. and Verspagen, B. (2009), Innovation studies: The emerging structure of a new scientific field, Research Policy 38(2): 218-33. doi.org/10.1016/j.respol.2008.12.006.

Godoe, H. (2012), Innovation theory, aesthetics, and science of the artificial after Herbert Simon, Journal of the Knowledge Economy 3(4): 372-88. doi.org/10.1007/s13132-0110055-6.

Gössling, T. and Rutten, R. (2007), Innovation in regions, European Planning Studies 15(2): 253-70. doi.org/10.1080/09654310601078788.

Guan, J. and Chen, K. (2012), Modeling the relative efficiency of national innovation systems, Research Policy 41(1): 102-15. doi.org/10.1016/j.respol.2011.07.001.

Guan, J. and Ma, N. (2003), Innovative capability and export performance of Chinese firms, Technovation 23(9): 737-47. doi.org/10.1016/S0166-4972(02)00013-5.

Huang, L. (2000), Approach to the main content of regional innovation systems, Science Research Management 21(2): 43-8.

Jacobsson, S. and Bergek, A. (2011), Innovation system analyses and sustainability transitions: Contributions and suggestions for research, Environmental Innovation \& Societal Transitions 1(1): 41-57. doi.org/10.1016/j.eist.2011.04.006. 
Jia, Y.N. (2001), Theory and analysis of regional creative milieus, [in Chinese], Areal Research \& Development 20(1): 5-8.

Li, W., Chang, J., Wang, M.J., Zhu, X.Y. and Jin, A.M. (2014), Innovation 3.0 and innovation ecosystems, [in Chinese], Studies in Science of Science 13(1): 39-63.

Martin, R. and Simmie, J. (2008), Path dependence and local innovation systems in cityregions, Innovation 10(2-3): 183-96. doi.org/10.5172/impp.453.10.2-3.183.

Motohashi, K. and Yun, X. (2007), China's innovation system reform and growing industry and science linkages, Research Policy 36(8): 1251-60. doi.org/10.1016/j.respol.2007.02.023.

Pekkarinen, S. and Harmaakorpi, V. (2006), Building regional innovation networks: The definition of an age business core process in a regional innovation system, Regional Studies 40: 401-13. doi.org/10.1080/00343400600725228.

Phan, P., Zhou, J. and Abrahamson, E. (2010), Creativity, innovation, and entrepreneurship in China, Social Science Electronic Publishing 6(2): 175-94. doi.org/10.1111/j.17408784.2010.00181.x.

Ronde, P. (2005), Innovations in regions: What does really matter?, Research Policy 34(8): 1150-72. doi.org/10.1016/j.respol.2005.03.011.

Simmie, J. (2003), Innovation and urban regions as national and international nodes for the transfer and sharing of knowledge, Regional Studies 37(6-7): 607-20. doi.org/10.1080/ 0034340032000108714 .

Sina Technology (2017), This document from the central government makes entrepreneurs very excited! Wang Jianlin says he is reassured, [in Chinese], China Business News, 30 September, available from: cj.sina.com.cn/article/detail/1650111241/424211 ?column=china\&ch=9.

Tian, Z.K., Zhao, X.J. and Tong H.Q. (2008), Comparison and assessment of China's technology innovation ability, [in Chinese], China Soft Science 7: 155-60.

Tong, J.S. (2003), The cooperative innovation system of industry, universities and research institutes and independent intellectual property, [in Chinese], China Soft Science (1): 113-16.

Wang, H.Q. and Hou, Y. (2017), Evaluation study on technological ability of high-tech industry in China, [in Chinese], Forum on Science and Technology in China 3: 58-63.

Wang, Z.H. and Liu, L. (2015), A comparative analysis of the national innovative evaluation indexes, [in Chinese], Science Research Management 1: 162-8.

Yin, W.H. and Zhang, Y.X. (2016), Analysis of independent innovation capability of industrial enterprises in China, [in Chinese], The World of Survey and Research 2: 36-47.

Zeng, G.P., Gou, Y.Z. and Liu, L. (2013), From innovation system to innovation ecosystem, [in Chinese], Studies in Science of Science 31(1): 4-12.

Zhou, B.X., Ding, Y.B. and Ren, C.M. (2007), Study on regional innovation systems structure model and operation mechanism, [in Chinese], China Soft Science (3): 135-8. 
This text is taken from The Chinese Economic Transformation: Views from Young Economists, edited by Ligang Song, Yixiao Zhou and Luke Hurst, published 2019 by ANU Press, The Australian National University, Canberra, Australia.

doi.org/10.22459/CET.2019.08 